\title{
Failure Analysis of Stainless Steel Bolt
}

\author{
Chun-Yan HU'1,2,3,a , Xin-Ling LIU ${ }^{1,2,3, b}$, Chun-Hu TAO ${ }^{1,2,3, c}$, Yu-Huai HE ${ }^{1,2,3, d}$ \\ ${ }^{1}$ Beijing Institute of Aeronautical Materials, Beijing 100095, China \\ ${ }^{2}$ Beijing Key Laboratory of Aeronautical Materials Testing and Evaluation, Beijing 100095, China \\ ${ }^{3}$ AVIC Failure Analysis Center, Beijing 100095, China \\ afachcy@163.com, ${ }^{b}$ liuxinling119@sohu.com, ${ }^{\mathrm{c}}$ chunhu.tao@biam.ac.cn, ${ }^{d}$ heyuhuai@163.com
}

Keywords: Bolt, Hydrogen Brittleness Fracture, Intensity, Assembly Stress, Hydrogen Content.

\begin{abstract}
Two stainless steel bolts of the motor components assembly fractured after serving for eight hours. The fracture feature and cause of the bolt were analyzed by appearance observation, micro and macro observation on fracture surfaces, metallographic examination, hardness testing. The results show that the fracture mode of the bolts is hydrogen brittleness fracture. The high intensity and biggish assembly stress led to the hydrogen brittleness fracture of the bolt. Meanwhile, there is a lack of dehydrogenation disposal after inactivating technology in the entire technological process. It is proposed that the intensity should be in the range of criterion requirements, and the dehydrogenation disposal should be disposed after passivation technology according to standard. What's more, the assembly stress should be controlled strictly to avoid early stage damage of bolt.
\end{abstract}

\section{Introduction}

Bolts are used as fastening pieces, which play a significant role in fixing and damping. In addition, the hydrogen brittleness fracture mode of bolts is always related to batch problem. Once failure faulty happen, it is dangerous for product safety and reliability, and enormous economic loss will happen [1-3]. The oil return flux was found to lower than required after driving device testing. As a result, two stainless steel bolts of the motor components assembly were found to have crack. The bolts experienced 8 hours' service, and the material is $1 \mathrm{Cr} 17 \mathrm{Ni} 2$ martensite stainless steel. The specific heat treatment processes were oil-quenched at $1020^{\circ} \mathrm{C}$ and tempered at $540 \pm 20^{\circ} \mathrm{C}$. The manufacturing processes are as follows: bar->roll screw->heat treatment->hydrogen nitrate passivating treatment->warehouse entry inspection. It is required that the hardness of the bolts be HRC30 38 and the tighten moment be $0.83 \sim 0.92 \mathrm{~N} \cdot \mathrm{m}$.

In order to determine the failure cause and to specify the preventive actions, appearance observation, micro and macro observation on fracture surfaces, metallographic examination and hardness testing were carried out.

\section{The Experimental Approach}

\section{Appearance and Macroscopic Observation}

The bolts appearance and fracture surface macroscopic morphologies were shown in Fig.1.

For describe simply, the two failed bolts classed as bolt A and B. The same batch bolt classed as bolt $\mathrm{C}$. The fracture position initiated at the intersection of the bolt and the net. The fracture surface was flat and crystal granular, and presented obvious reflecting small facets. No obvious plastic yield was seen at the region. 

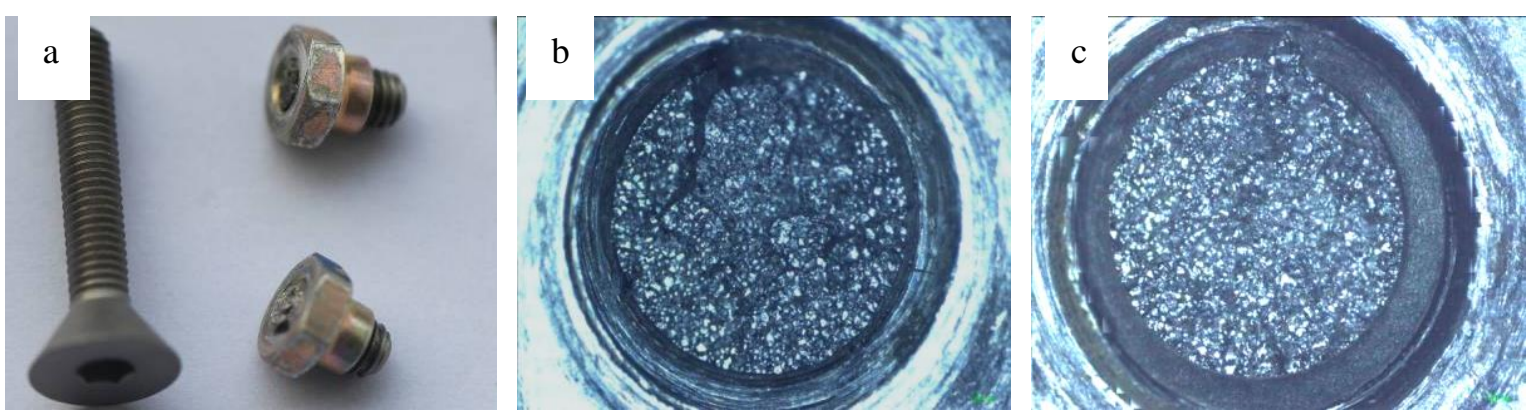

Fig.1 Appearance and macro-morphology of the fracture surface of the failed bolts (a)Appearance and fracture position of the bolt; (b)Macro-morphology of the fracture surface of the failed bolt A; (c)Macro-morphology of the fracture surface of the failed bolt B

\section{Micro Observation on Fracture Surface}

The microscopic morphologies of the fracture surface and the screw face of the failed bolt A were shown in Fig.2.

The fracture surface can be divided to three regions. Intergranular and tearing edges feature can be seen at zone 1. High-magnification appearance showed that quasi-cleavage at zone 2. Typical Dimples feature existed at the final fracture region(zone 3). Obvious extruding trace can be seen at the screw face.

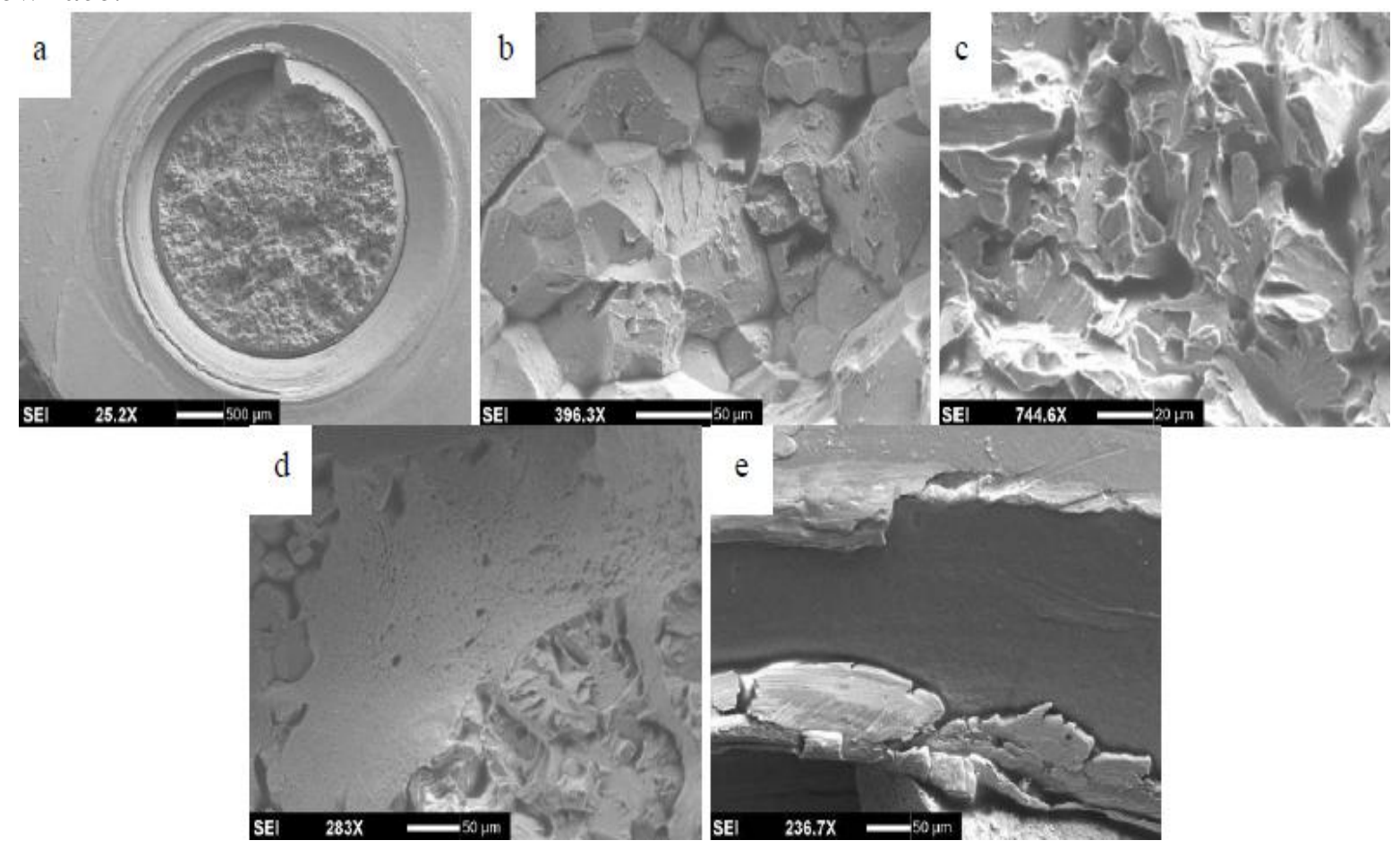

Fig.2 SEM morphology of the fracture surface of the failed bolt A

(a)Low-powered morphology of the fracture surface; (b)ntergranular feature of zone;

(c)Quasi-cleavage feature of zone 2; (d)Dimples feature of zone 3; (e)Extrusion impression of screw surface

The microscopic morphologies of the fracture surface and the screw face of the failed bolt B were shown in Fig.3.

The fracture surface can be divided to two regions. High-magnification appearance showed that quasi-cleavage and small quantity dimples feature existed at the center region. Intergranular and tearing edges feature can be seen at the rest regions. Obvious extruding trace can be seen at the screw face. 

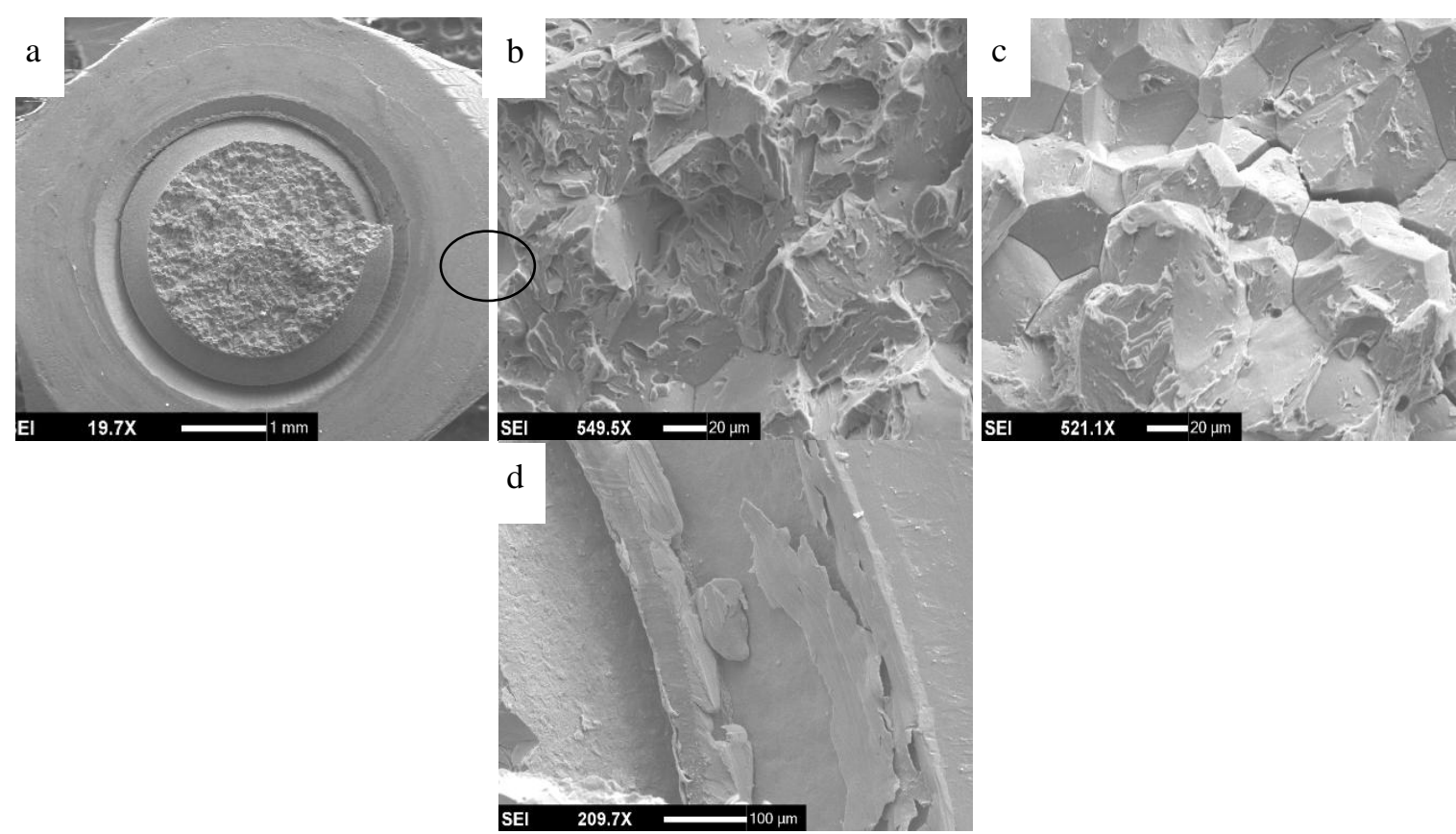

$521.1 \mathrm{X}$

Fig.3 SEM morphology of the fracture surface of the failed bolt B

(a)Low-powered morphology of the fracture surface; (b)Intergranular feature; (c)Quasi-cleavage and dimples feature of center; (d)Extrusion impression of screw surface

\section{Metallographic Examination}

Metallographic specimens perpendicular to the fracture surface of bolt $\mathrm{A}$ and bolt $\mathrm{C}$ were cut down. The results show that the microscopic structure of bolt $\mathrm{A}$ and bolt $\mathrm{C}$ are composed of martensite, shown in Fig.4.
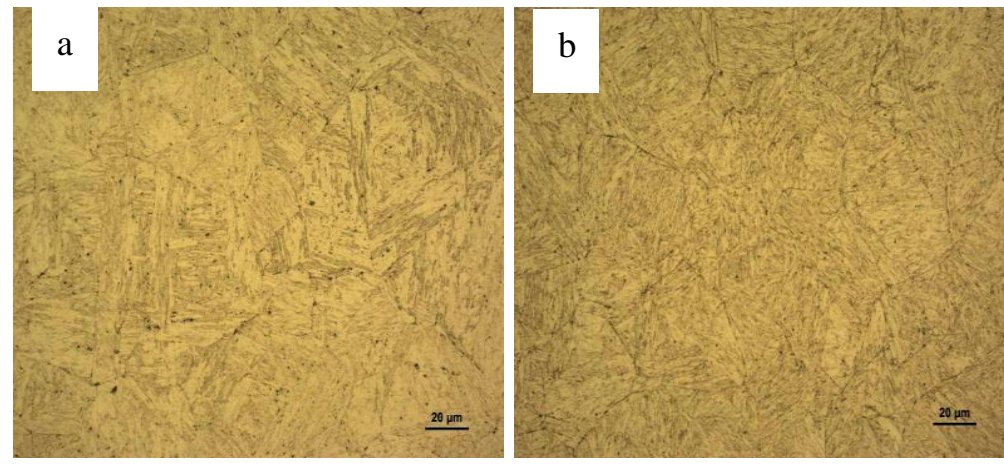

Fig.4 Metallographic microstructure of the bolts

(a) Metallographic microstructure of the failed bolt A; (b) Metallographic microstructure of the bolt C

\section{Hardness Testing}

Samples perpendicular to the fracture surface were cut down to perform hardness testing. HV were converted to HRC according to GB/T1172-1999; the results were shown in table 1. According to table 1, the hardness is higher than HRC30 38 required.

Tab. 1 Hardness testing results

\begin{tabular}{cccccccc}
\hline Sample & 1 & 2 & 3 & 4 & 5 & 6 & $\begin{array}{c}\text { Average } \\
\text { value }\end{array}$ \\
\hline Blot A & 413.0 & 419.4 & 420.9 & 416.2 & 409.8 & 425.9 & 417.5 \\
\hline Blot C & 427.2 & 419.4 & 425.9 & 414.6 & 413.0 & 413.0 & 418.9 \\
\hline
\end{tabular}




\section{Results and Discussion}

\section{Analysis of Failure Mode}

According to the intergranular and tearing edges character at the fracture surface, it can be assumed that the fracture mode of the bolts is hydrogen brittleness fracture. In addition, according to the quasi-cleavage and dimples at the fracture surface of the artificially broken bolt, the temper brittleness failure mode can be eliminated.

\section{Analysis of Failure Cause}

In generall, the hydrogen brittleness fracture are relative with material intensity, hydrogen content and tensile stress [4-6]. The following parts were analyzed from these aspects. Firstly, the average hardness of bolts is about HRC 43, which higher than HRC30 38 required, and change to tensile extension is about $1339 \mathrm{MPa}$. In addition, the material intensity is always related to the sensitivity of hydrogen brittleness fracture [7].

Secondly, the hydrogen content in bolts mostly rooted in the passivation technology. In the passivation process, the superficial oxidate of metal generated chemistry reaction with acid. However, the partial metal also reacted with acid, which created the hydrogen may enter into metal. It is found that the passivation fluid component, passivation time and alloy component can affect the infiltration capacity of the hydrogen. Because of the hydrogen be prone to gather at the root radius of screw, the hydrogen content may relatively higher. Bolt's material had higher sensitivity of hydrogen brittleness, which did not meet technical requirement. According to HB/Z 318-1998, steel accessories were required dehydrogenation treatment after passivation technology. It is defined that the tensile extension of steel accessories higher than the requirement $(\geq 1050 \mathrm{MPa})$ have to proceed dehydrogenation treatment.

Furthermore, according to the obvious extruding trace at the screw face, it can be assumed that the assembly stress of bolts is biggish and may cause early stage damage. In addition, the fracture position initiated at the root radius of first screw, which had wider stress concentration and be propitious to hydrogenous diffuse. With the stress effect, hydrogen atomic which located at the lattice gap may diffuse to the position of stress concentration. When the dislocation lines were fixed, the basal body would become fragile, because of the interaction of the hydrogen atomic and dislocation [8].

In order to enhance the application reliability of bolts and to prevent re-occurrence of such failure, it is suggested that the intensity should be in the range of criterion requirements, and the dehydrogenation disposal should be disposed after passivation technology according to standard. What's more, the assembly stress should be controlled strictly to avoid early stage damage of bolts.

\section{Conclusions}

Three summary observations for u-shaped bolts failure can be made based on the results presented here.

(1) The failure mode of the bolts is hydrogen brittleness fracture.

(2) The higher intensity and biggish assembly stress, which were the main causes for the bolts fracture.

(3) It is suggested that the intensity should be in the range of criterion requirements, and the dehydrogenation disposal should be disposed after passivation technology according to standard. What's more, the assembly stress should be controlled strictly to avoid early stage damage of bolt.

\section{References}

[1] C.K. Liu, J.X. Zang, B. Zhang. Failure analysis of 30CrMnSiA bolt fracture, Failure Analysis and Prevention. 3(2008)42-47. 
[2] C.G. Li, Y. Yu. Failure analysis on fracture of $30 \mathrm{CrMnSiNi2A}$ steel bolt, Materials for Mechanical Engineering. 34(2010)75-78.

[3] X. Yan, N. Li. Analysis on surface cracks of self-made bolt, Failure Analysis and Prevention. 5(2010)243-246.

[4] B. Liu. The fracture mechanism of hydrogen embrittlement in 30CrMnSiA high strength steel, Materials for Mechanical Engineering. 25(2001)18-21.

[5] W.Y. Zhu, L.J. Qiao, Q.Z. Chen, et al. Fracture and environmental fracture, Beijing, 2000.

[6] X.Y. Sun. Study of hydrogen brittleness of bolt, Aerospace Standardizition. 2(2007)1-9.

[7] G.R. Fu, Z. Zhang. Failure analysis technology, Physical testing and chemical analysis part A: physical testing. 41(2005)212-216.

[8] D. Zhang, P.D. Zhong, C.H. Tao, et al. Failure analysis, Beijing, 2004. 